\title{
Inversion of Dopamine Responses in Striatal Medium Spiny Neurons and Involuntary Movements
}

\author{
Li Liang, Mahlon R. DeLong, and Stella M. Papa \\ Department of Neurology, School of Medicine, Emory University, Atlanta, Georgia 30322
}

\begin{abstract}
Dopamine influence in the striatum is essential to motor behavior and may lead to involuntary movements in pathologic conditions. The basic mechanisms lie in differential dopamine responses of medium spiny neurons (MSNs) contributing to striatal output pathways. The relationship between striatal discharge and mobility is thus critical to understanding the actions of dopamine. Using extracellular recordings in severely parkinsonian monkeys, we examined the activity changes of MSNs during different levels of dopamine stimulation. The activity of single MSNs was recorded continuously throughout conditions of parkinsonian disability, its reversal, and the exhibition of involuntary movements after levodopa administration. Parkinsonian disability was associated with robust and widely distributed increases of MSN firing. In the parkinsonian state, dopamine influx produced both increases and decreases in the discharge rate of MSNs. Furthermore, in contrast to the expected net reduction of activity, dopamine-induced recovery of mobility occurred with predominant further increases of neuronal activity. In contrast, involuntary movements were associated with a distinctive inversion of the dopamine responses. The activity increases and decreases associated with the recovery of mobility were subsequently inverted in a number of neurons, and these bidirectional changes created large differences of discharge across MSNs. Thus, a markedly dysregulated state of striatal activity develops after chronic dopamine denervation and, in such a state of MSN activity, dopamine induces altered and disproportionate responses. These findings point to the fundamental role of dopamine-mediated balance of striatal outputs for normal movement.
\end{abstract}

Key words: striatum; electrophysiology; dopamine; Parkinson's disease; involuntary movements; dyskinesias

\section{Introduction}

Dopamine input to the striatum plays a key role in the control of motor function, and its depletion is the primary cause of motor disability in Parkinson's disease (PD). Replacement therapy restores mobility, but it may also lead to development of involuntary movements (dyskinesias) through unclear mechanisms. Acting through G-protein-coupled receptors, dopamine alters voltage-dependent ion channels and thereby modulates MSN membrane excitability (Surmeier et al., 1995; Nicola et al., 2000). Depolarization requires glutamatergic inputs, but action potential firing depends on the state of membrane potentials that typically shift from "up" (depolarized) and "down" (hyperpolarized) states under the influence of dopamine (Wilson and Kawaguchi, 1996; Plenz and Kitai, 1998; West and Grace, 2002). This modulatory action is differentially mediated through $\mathrm{D}_{1}$ (excitatory) and $\mathrm{D}_{2}$ (inhibitory) receptors (Kitai and Surmeier, 1993; West et al., 2003), which are mostly segregated in the direct and indirect striatal output pathways, respectively (Gerfen, 1992; Surmeier et al., 1996). According to current models (Albin et al.,

Received Nov. 26, 2007; revised June 5, 2008; accepted June 17, 2008.

This work was supported by National Institutes of Health Grant NS 045962 to S.M.P. We thank Drs. Mandar Jog and Dieter Jaeger for helpful comments on this manuscript. The extended use of severely parkinsonian monkeys was possible because of the dedicated assistance of Jessica Whithear and the veterinary staff of the Yerkes National Primate Research Center.

Correspondence should be addressed to Dr. Stella M. Papa, Department of Neurology, School of Medicine, Emory University, 6000 WMRB, 101 Woodruff Circle, Atlanta, GA 30322. E-mail: spapa@emory.edu.

D0I:10.1523/JNEUROSCI.1176-08.2008

Copyright $\odot 2008$ Society for Neuroscience $\quad$ 0270-6474/08/287537-11\$15.00/0
1989; DeLong, 1990), after dopamine depletion the excitability of MSNs is decreased in the direct pathway and increased in the indirect pathway. However, sustained denervation may lead to functional changes affecting complex dopamine receptormediated effects (Surmeier et al., 1995; Cepeda et al., 1998), and to morphological changes of glutamatergic synapses on selective MSN subpopulations (Day et al., 2006). In addition, based on receptor regulation and other signaling changes, it has been proposed that dyskinesias result from the imbalance of dopamine regulatory actions (Chase et al., 1996; Onn et al., 2000). Moreover, long-term depression, a dopamine-mediated mechanism of synaptic plasticity (Graybiel et al., 1994; Calabresi et al., 2000; Wang et al., 2006), can be altered selectively in the indirect pathway (Kreitzer and Malenka, 2007), or the direct pathway in relation to dyskinesias (Picconi et al., 2003). However, with most data derived from recordings in vitro or in anesthetized animals, the differential action of dopamine on the MSN excitability of segregated pathways has yet to be correlated with motor behaviors in PD.

The low spontaneous activity of MSNs (Crutcher and DeLong, 1984; Kimura, 1992), which are frequently silent if unrelated to a task performance, complicates thorough recordings in normal animals. Dopamine depletion, however, may lead to higher spontaneous activity of MSNs (Ingham et al., 1998; Gubellini et al., 2002). Thus, we used two severely parkinsonian rhesus monkeys to complete recordings of single MSNs during prolonged intervals and to identify the firing changes associated 
with different motor states after levodopa administration (see Fig. 1a). Our first hypothesis, congruent with current models, was that dopamine denervation results in activity decreases and increases in MSN subpopulations corresponding to neurons of the direct $\left(D_{1}\right.$ receptors $)$ and indirect $\left(D_{2}\right.$ receptors $)$ pathways, respectively. The second hypothesis was that the effects of levodopa administration are mediated by different dopamine actions on the segregated MSN subpopulations. Hence, we expected to find equivalent numbers of activity increases and decreases associated with reversal of parkinsonian symptoms. Additionally, imbalance of dopamine actions between subpopulations of MSNs was predicted to correlate with dyskinesias, as currently modeled.

\section{Materials and Methods}

Animal preparation and behavior. Two adult female rhesus monkeys (Macaca mulatta, 5-7 kg) were used in this study, which was conducted in accordance with the National Institutes of Health Guide for the Care and Use of Laboratory Animals (1996). Monkeys L and U were rendered parkinsonian by systemic administration of 1-methyl-4-phenyl-1,2,3,6tetrahydropyridine (MPTP) more than 1 year before this study, and met the criteria for chronic, severe, and late-stage parkinsonism with exhibition of levodopa-induced dyskinesias (dyskinesias are usually absent in mildly parkinsonian rhesus monkeys). To induce an extensive lesion, MPTP $(0.3-0.8 \mathrm{mg} / \mathrm{kg}$, i.v.) was injected at weekly intervals during several months. Motor disability was assessed periodically with a standardized Motor Disability Scale (MDS) for MPTP-treated monkeys (Papa and Chase, 1996) until stabilization at 18 or higher motor disability scores (see levodopa tests below). Subsequently, monkeys received oral carbidopa/levodopa daily until development of marked and consistent dyskinesias (part II of the MDS). Both monkeys had a severe and chronic parkinsonism with stable levodopa-induced dyskinesias.

Doses of levodopa methyl ester plus benserazide given subcutaneously to elicit reproducible responses in successive recording sessions were screened in each monkey. Two doses were selected: (1) dyskinetic doses (50-75 mg, s.c.) that consistently produced peak-dose dyskinesias of mild to moderate intensity for compatibility with the monkey's restraint in the primate chair during recordings, and (2) nondyskinetic doses (30-40 mg, s.c.) that did not elicit dyskinesias but were sufficient to induce an antiparkinsonian response measurable with the standardized scale. Because severely parkinsonian monkeys have variable responses to levodopa and, similar to patients, a narrow therapeutic window, we selected for this study animals that did not have an "all or none" response. Thus, lower doses of levodopa that induced measurable responses could be identified for these monkeys. Levodopa responses were reproduced on repeated tests (a minimum of 3 ). Because recordings in each monkey were maintained for $>12$ months, consistency of levodopa responses was tested periodically in the monkey's home cage to allow full scoring using the MDS. Higher doses of levodopa (50-75 mg, s.c.) resulted in the following total motor disability scores (averages of repeated tests): $21 \pm$ 2, "Off" state (parkinsonian disability) at baseline; and 7.4 \pm 0.8 , "On" state (reversal of disability) at the peak of the levodopa effect (mean \pm SEM). Lower doses of levodopa (30-40 mg, s.c.) tests resulted in the following total motor disability scores: $19.7 \pm 1.9$, Off state at baseline; and $11.1 \pm 1$, On state at peak of levodopa effect. Noticeably, the baseline motor disability was in the highest range for rhesus monkeys (18-23), and thus it correlates with severe dopamine depletion. Dyskinesias scores at the peak of levodopa effects were as follows: $6.4 \pm 0.6$ with higher doses, and 0 with lower doses. Because of the prolonged survival of the animals in this study and their advanced disability, regular oral treatment was used for maintenance $(25-50 \mathrm{mg} / \mathrm{d})$.

After behavioral testing, monkeys were surgically implanted with a recording chamber and a head-holding device under general anesthesia with isoflurane and following standard procedures. The recording chamber ( $18 \mathrm{~mm}$ inner diameter) was placed at a $20^{\circ}$ angle in the sagittal plane and centered to include basal ganglia regions for mapping purposes using stereotaxic coordinates from the monkey atlas (Winters et al., 1969). This position allowed microelectrodes to follow a dorsorostral to ventrocaudal trajectory.
On experiment days, oral levodopa treatment was withdrawn, and monkeys were transferred to the primate chair in the mornings, while parkinsonian disability was at its highest score. A butterfly needle connected to a tube for distal access was placed under the skin in the back over the interscapular area in preparation for levodopa injections. Motor behavior was continuously monitored through videotaping. The advanced parkinsonism in these monkeys (severe disability and significant dyskinesias) produced a profound and invariable Off state through successive experiment days. Both levodopa doses produced an On state that was identified clearly in recording sessions despite slightly higher disability scores with the lower doses. The onset of the On state (Fig. 1a), usually $20 \mathrm{~min}$ after the subcutaneous levodopa injection, was recognized typically by rapid movements of the eyes, increased blinking, yawning, and stretching of the legs (all marked as voluntary movements or contamination if they introduced changes in recordings). Motor disability scores remained low until re-entering the Off state (110 min after the injection). Dyskinetic doses of levodopa usually produced mild peak-dose dyskinesias (total scores, $<7$ points) (see Fig. $1 a$ ), predominantly as choreodystonic movements in the leg (one animal had clear oral dyskinesias too). Dyskinesias began $\sim 15 \mathrm{~min}$ after turning On and lasted for $40-50 \mathrm{~min}$. Dyskinesias were distinguished from voluntary movements because of typical features, i.e., stereotyped or repetitive purposeless movements. Data were collected for 9-12 $\mathrm{min}$ in each state. To ensure the transition to the next state (the critical condition for storing neural data), a minimum of 3-5 min of behavioral changes was required. In experiments with lower doses of levodopa, the onset of the On state could be detected clearly by similar signs as with higher doses, although animals were less active (higher disability scores). Lower doses produced a motor response without dyskinesias in any segment of the body, and the interval for the "On-without-dyskinesias" state was taken by matching the timing of the "On-with-dyskinesias" state in high-dose experiments (Papa et al., 1999). Longer time frames were recorded in the On-without-dyskinesias state to avoid missing delayed changes caused by lower doses.

Electrophysiology and data analysis. Approximately one of three recording sessions could be completed because of frequent inability to isolate the neuron for the period (50-60 min) necessary for continuous recording while the monkey passed through different motor states. Most aborted experiments occurred because stable spikes were lost because of excessive movement at the onset of the On state or during dyskinesias. Electrode tracks through striatum showed a variable number of spontaneously active neurons, from 1 to 4.5 cells $/ \mathrm{mm}$ (mean, $2.7 \pm 0.15$ ). The number was lower as tracks were made in more lateral areas of putamen. Units that could be classified as interneurons by direct on-line assessment were not selected for data storing and continuation of the experiment. Only experiments with complete series of data for all motor states (see Fig. 1a) were included in the analysis. Off-line, units were thoroughly examined for separation of interneurons according to established criteria (Kimura, 1986, 1992; Aosaki et al., 1994; Raz et al., 1996) (supplemental text, Classification of units, and supplemental Fig. 1, available at www.jneurosci.org as supplemental material). The total number of neurons recorded in experiments of both doses of levodopa was 223. Thirty-five units (16\%) were excluded from analyses after reassessment off-line. Thus, 188 units (84\%) were classified as MSNs and fully analyzed in this study.

Tungsten microelectrodes (impedance, 9-12 M $\Omega$ at $1000 \mathrm{~Hz}$ and $0.3-2 \mathrm{M} \Omega$ after reconditioning) for recording of single-cell activity were lowered with a hydraulic microdrive through a guide cannula to the striatum. Signals were amplified and bandpass filtered with a $100-8000$ $\mathrm{Hz}$ four-pole filter (multichannel acquisition processor; Plexon). Programmable filter and gain settings varied with experimental conditions (total gain, 5-20 K). Data were acquired at the sampling rate of $20 \mathrm{kHz}$, and a real-time spike-sorting analysis was used on-line to evaluate the quality of unit isolation throughout the session. Different algorithms of spike sorting using principal component analysis could be selected depending on the data (Plexon). Raw data were stored, and definitive spike sorting was performed off-line. A separate channel was used for inserting event marks (event acquisition channel) to indicate behavioral parameters, such as drug injection and the transition to different motor states. Event marks were aligned with neuronal activity by time stamps. 
Off-line analysis of stored data began with examination of the spike train for movement artifacts or investigator's marks (event marks) indicating behavioral changes. The animal was continuously monitored to detect obvious movement and introduce marks in the recordings. Appropriate segments corresponding to the monkey at rest in each state were compiled for spike sorting and, because $\sim 9$ min of recording were usually stored, a minimum of 3 min was finally analyzed in each state. Data from one or occasionally two units were obtained after spike sorting (attempts to use multiple electrodes to increase data collection were unfruitful because of difficulties holding stable striatal units on separate electrodes during the transition to motor states). After sorting, only units classified as MSNs were included in the study. Units excluded were classified as interneurons (fast-firing interneurons and tonically active neurons; $4 \%$ ), uncertain location with relocation of tracks after histology (3.6\%), or unclear classification (8.1\%).

MSN data analyses. Data were analyzed initially by matching waveforms to confirm that activity of the same unit with stationary spiking was processed in each state. Subsequently, a burst detection algorithm (Kaneoke and Vitek, 1996) was used with a custom-made software to separate "bursty" and "nonbursty" MSNs. Detected bursts had consistent spike waveforms with minimal amplitude changes after the spikesorting algorithm was applied for all intervals of the spike train (three motor states). In each unit, firing rates were determined with rate meters ( $1 \mathrm{~s} / \mathrm{bin}$ width; Neuroexplorer) constructed on the entire spike train of 3 min or longer in each state. The spike rates in each individual neuron were converted to $z$ scores: $\mathrm{FF}_{\mathrm{P}} \pm \mathrm{FF}_{\mathrm{N}} / \mathrm{SE}_{\mathrm{P}}$, where $\mathrm{FF}_{\mathrm{P}}$ is the mean firing frequency at baseline taken from the "previous state," $\mathrm{FF}_{\mathrm{N}}$ is the mean firing frequency corresponding to the transition to the "next state," and $\mathrm{SE}_{\mathrm{P}}$ is the $\mathrm{SE}$ of the mean firing frequency of the previous state (Barnes et al., 2005). For acceptance, limits were set as $99 \%$ or $\alpha / 2=0.005$, and, therefore, differences were taken at $\mathrm{FF}_{\mathrm{P}} \pm \mathrm{FF}_{\mathrm{N}}>\mathrm{SE}_{\mathrm{P}} \times Z_{\alpha / 2=0.005}$. Subsequently, differences of discharge rate in each motor state, Off, On, and On with or without dyskinesias for each individual neuron were compared using ANOVA for repeated measures followed by the post hoc Bonferroni's test when the ANOVA $F$ value indicated significance taken at $p<0.01$ (large sample sizes of 180 or higher number of frequency values with bins of $1 \mathrm{~s}$ ).

Data from units with significant rate changes in the transition to motor states were also compared between the two sites of recordings: the putamen and caudate. Units were grouped according to their rate changes from Off to On and to On-with-dyskinesias states in each region, and differences were analyzed with factorial ANOVAs (recording regions and groups of rate changes) for repeated measures (firing rates in motor states). In addition, the total magnitude of rate changes through the three motor states was calculated. Because in units with bidirectional changes of rate (e.g., increase followed by decrease) the difference from the Off to the On state was often eliminated at the On-with-dyskinesias state, we calculated the algebraic addition and subtraction of rate differences for unidirectional and bidirectional responses, respectively. This calculation was selected to express the total magnitude of change with positive or negative signs, which indicate the direction of the primary rate change from the Off to the On state for group identification. Thus, in each neuron, the total value of rate differences was obtained as follows: $\left(\mathrm{FF}_{n}-\right.$ $\left.\mathrm{FF}_{\mathrm{f}}\right)+\left(\mathrm{FF}_{\mathrm{d}}-\mathrm{FF}_{\mathrm{n}}\right)$ for unidirectional responses and $\left(\mathrm{FF}_{\mathrm{n}}-\mathrm{FF}_{\mathrm{f}}\right)-\left(\mathrm{FF}_{\mathrm{d}}\right.$ $-\mathrm{FF}_{\mathrm{n}}$ ) for bidirectional responses, where $\mathrm{FF}_{\mathrm{n}}$ is the mean firing frequency in the On state, $\mathrm{FF}_{\mathrm{f}}$ is the mean firing frequency in the Off state, and $\mathrm{FF}_{\mathrm{d}}$ is the mean firing frequency in the On-with-dyskinesias state. The total magnitude of rate changes in each group of responses in the putamen and caudate were compared with factorial ANOVA.

Histology. At the end of the study, monkeys were killed under deep anesthesia for histological examination of electrode tracks. For the purpose of track visualization, an electrode penetration with known coordinates from previous recordings was made just before the animals were killed to produce a visible scar. The brain was blocked, postfixed with paraformaldehyde, and cut in 30-50 $\mu \mathrm{m}$ sections. Nissl staining of brain sections showed the electrode track passing through targeted anatomical structures. The remaining areas of recording were confirmed in reference to coordinates of the visualized electrode track using the monkey brain atlas (Winters et al., 1969), as shown in Figure $1 b$. a
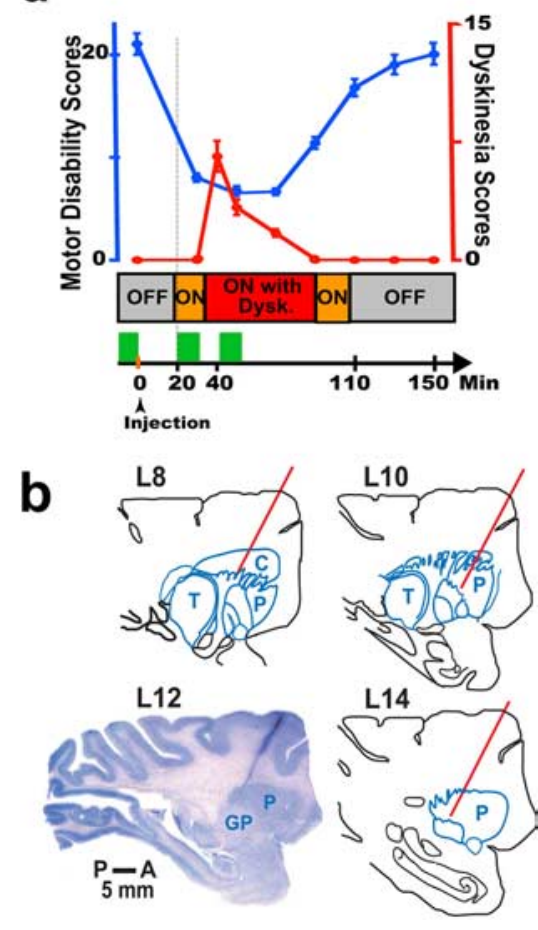

Figure 1. Motor behavior, timetable of experiments, and recording sites. $\boldsymbol{a}$, The top graph shows motor disability (blue curve; left $y$-axis) and dyskinesias (Dysk.; red curve; right $y$-axis) that were scored with the standardized motor disability scale (data points represent the mean, and error bars denote SEM). The middle stack bar shows the monkey's transition to motor states during the experiment (Off, On, and On with dyskinesias) (see Materials and Methods). At the bottom, the sequence of procedures is represented by a horizontal arrow that is plotted synchronously with the top graph and the middle stack bar, as indicated by the vertical line. Green blocks denote collection of neural data (9-12 min) starting in the Off state, and again in the 0 n state (usually $20 \mathrm{~min}$ after the subcutaneous injection of levodopa), and finally during the peak of dyskinesias (usually after $40 \mathrm{~min}$ from levodopa injection). Experiments of lower doses of levodopa that did not induce dyskinesias (not plotted for clarity) followed the same timetable and neural data collection in each state. $\boldsymbol{b}$, The Nissl-stained section of the monkey brain shows the electrode track of a brain penetration made just before the animal was killed. To show one of the recording sites, the electrode track was placed at the posterior and lateral area of the putamen. The section matched the anatomy of lateral 12 in the sagittal plane of the monkey atlas (L12). The position of other electrode penetrations was confirmed with reference to the coordinates of the electrode track in $L 12$, and they are represented by red lines in the drawings of corresponding sections according to the atlas. The bar shows the scale from posterior (P) to anterior (A). C, Caudate; P, putamen; GP, globus pallidus; T, thalamus.

\section{Results}

\section{Parkinsonian baseline activity}

The activity of 188 MSNs (monkeys L and U) was collected for analysis during $>400$ sessions of single-cell recording (see Materials and Methods). Analyzed data in each single-cell recording were taken in the absence of obvious movement of any body part. The sensorimotor area of the striatum (Crutcher and DeLong, 1984; Alexander and DeLong, 1985) (posterolateral region of the putamen), as located by electrophysiological mapping of basal ganglia, typically exhibited lower spontaneous activity than other striatal regions. In this area of putamen (Fig. $1 b$ ), data from 96 MSNs were collected; also, data from 92 MSNs were collected in the middle portion of the caudate nucleus (less involved in sensorimotor function) (Alexander and DeLong, 1985). Putamen and caudate data were analyzed separately and correlated subsequently. In experiments with dyskinetic doses of levodopa, 140 neurons were recorded, 73 in the putamen and 67 in the caudate ( 86 for $\mathrm{L}$ and 54 for $\mathrm{U}$, each proportionally distributed in both 

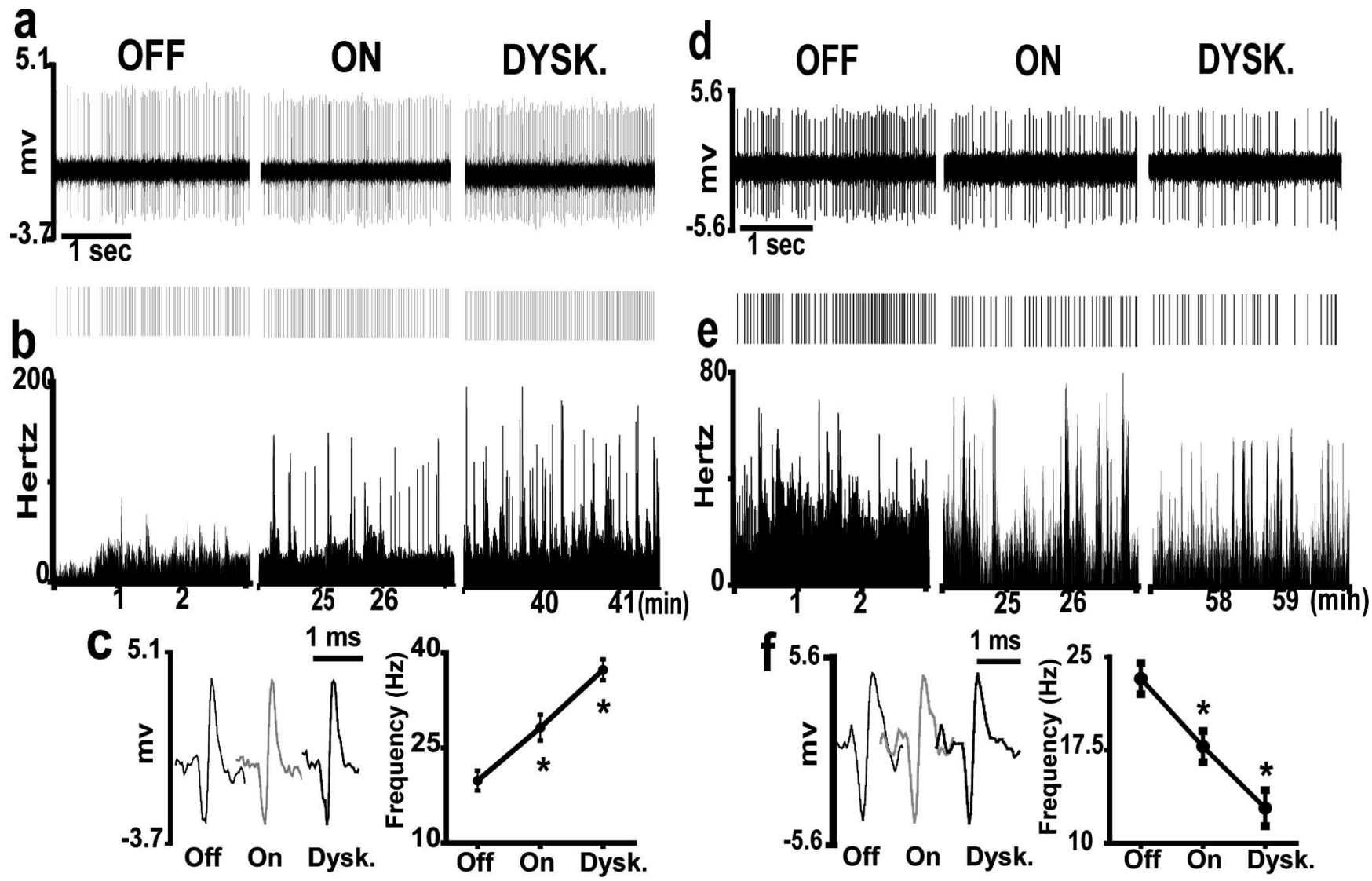

Figure 2. Examples of activity changes of MSNs responding with unidirectional changes of frequency. Two examples of single neuronal activity recorded in the putamen continuously while the monkey passed from 0 ff to 0 n and to 0 -with-dyskinesias (Dysk.) states are shown in $\boldsymbol{a}$ - c for one unit and $\boldsymbol{d}$ - $\boldsymbol{f}$ for another unit. $\boldsymbol{a}$, Selected spike trains of the same unit during each motor state and their matching rasters below. The segments shown in the figure were taken from the original file (usually $180 \mathrm{~s}$ in each state) and are parts of the data used in the final analysis. $\boldsymbol{b}$, Rate meters. The graphs show the rate meters for the corresponding neuron presented above $(\boldsymbol{a})$ but for the time analyzed in each motor state $(\sim 3 \mathrm{~min}$; bin width, $1 \mathrm{~s})$. $\boldsymbol{c}$, Left, Plot of waveforms of the isolated neuron (negative deflection downward) during the transition to each motor state. Notice the match of waveforms demonstrating data collection from the same neuron throughout the experiment. Right, Graph of averaged rates in each state from the same neuron as above ( $20 \pm 0.5 \mathrm{~Hz}$ in $0 f f, 28 \pm 0.7 \mathrm{~Hz}$ in $0 \mathrm{n}$, and $37 \pm 0.6 \mathrm{~Hz}$ in the $0 \mathrm{n}$-with-dyskinesias state). Description and conventions for $\boldsymbol{d}-\boldsymbol{f}$ are the same as in $\boldsymbol{a}$ - $\boldsymbol{c . f}$, The averaged rates are as follows: $23 \pm 0.4 \mathrm{~Hz}$ in $0 \mathrm{ff}, 18 \pm 0.4 \mathrm{~Hz}$ in $0 \mathrm{n}$, and $13 \pm 0.5 \mathrm{~Hz}$ in the $0 \mathrm{n}$-with-dyskinesias state. ${ }^{*} p<0.0001$ versus the previous state (error bars denote SEM).

areas); with nondyskinetic doses, 48 neurons were recorded, 23 in the putamen and 25 in the caudate.

Firing frequencies in the parkinsonian state (Off state) in all neurons (188) ranged from 2.7 to $52 \mathrm{~Hz}$ and averaged $28.2 \pm 1.5$ $\mathrm{Hz}$ in the putamen $(96)$ and $23.5 \pm 1.3 \mathrm{~Hz}$ in the caudate (92). These frequencies (Figs. 2, 3, Off state) represent major increases compared with those classically described in normal conditions $(0.5-2 \mathrm{~Hz})$. Also, increased frequencies were found in both the putamen and caudate. Units were sampled randomly and not based on their firing frequencies. A point that needs especial consideration is whether or not units were sampled only from a particular subpopulation of neurons. In this regard, it is expected that direct projection neurons reduce their firing after dopamine depletion because of absence of $\mathrm{D}_{1}$ receptor-mediated excitatory influences. These units could have been silent $(<0.5-2 \mathrm{~Hz})$ and thus not sampled in these recordings. Then, most recorded units would have been indirect $\mathrm{D}_{2}$ receptor-bearing neurons. However, a large proportion of the recorded neurons (64\% of total) responded to levodopa administration with increases of activity (see below, Dopamine stimulation) that indicated the predominant $D_{1}$ receptor-mediated response typical of the direct output pathway. Analysis of dopamine responses (see below) allowed us to infer that sampled MSNs were indistinctively distributed among subpopulations of segregated projections (units express- ing increases and decreases of firing that correspond to predominantly $\mathrm{D}_{1}$ - and $\mathrm{D}_{2}$-mediated responses of the direct and indirect output pathway, respectively). Comparisons of baseline firing rates between both groups of units showed a small but significant difference (Table 1). This difference reflects the differential effects of dopamine depletion on predominantly $D_{1}$ and $D_{2}$ receptor-bearing units, i.e., higher baseline rates in units with dopamine $\mathrm{D}_{2}$-mediated activity decreases, and lower baseline rates in units with dopamine $\mathrm{D}_{1}$-mediated activity increases. In summary, after dopamine denervation, MSN baseline rates are markedly increased independently of the dopamine receptor subpopulation and thus the output pathway, although these higher rates still differ between the two subpopulations. These results contrast with the expected reduction or silencing of activity in the direct output pathway after dopaminergic lesions. It is noteworthy that, as opposed to the effects of acute dopamine depletion (Costa et al., 2006), increased firing rates across MSNs developed in our setting of chronic dopamine deficiency, which is presumably associated with the development of compensatory mechanisms and long-term plastic changes.

MSNs more commonly discharged in an irregular tonic/phasic manner (nonbursty neurons), whereas a smaller proportion of units fired with variable bursts intermixed with periods of irregular spiking (bursty neurons). This burst activity in the Off 

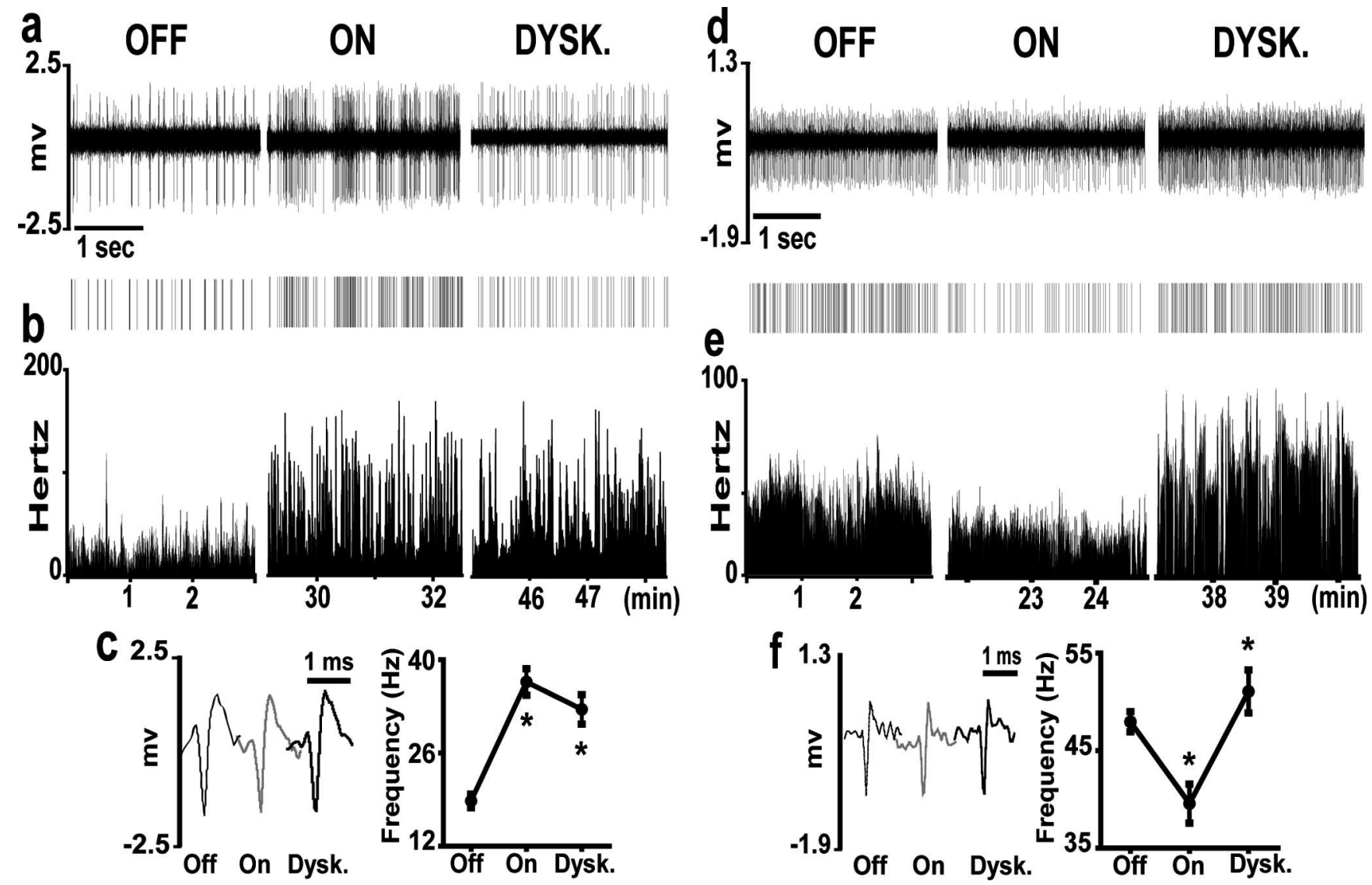

Figure 3. Examples of activity changes of MSNs responding with bidirectional changes of frequency. $\boldsymbol{a}-\boldsymbol{f}$, Descriptions and conventions are the same as in Figure 2 . $\boldsymbol{c}$, The averaged rates are as follows: $19 \pm 0.4 \mathrm{~Hz}$ in $0 \mathrm{ff}, 37 \pm 0.7 \mathrm{~Hz}$ in $0 \mathrm{n}$, and $32 \pm 0.7 \mathrm{~Hz}$ in the $0 \mathrm{n}$-with-dyskinesias state. $f$, The averaged rates are as follows: $48 \pm 0.5 \mathrm{~Hz}$ in $0 \mathrm{ff}, 39 \pm 0.8 \mathrm{~Hz}$ in $0 \mathrm{n}$, and $51 \pm 0.8 \mathrm{~Hz}$ in the On-with-dyskinesias state. ${ }^{*} p<0.0001$ versus the previous state (error bars denote SEM).

Table 1. MSN firing rates in the parkinsonian state (Off state)

\begin{tabular}{lll}
\hline Separation by dopamine responses & Percentage of units $^{a}$ & Baseline rate $(\mathrm{Hz})$ \\
\hline Increased activity $\left(D_{1}\right)$ & $64 \%$ & $25 \pm 1.3$ \\
Decreased activity $\left(D_{2}\right)$ & $34 \%$ & $33 \pm 1.7^{*}$
\end{tabular}

All units were separated according to dopamine responses, as increased or decreased activity $(n=140)$.

${ }^{a}$ The proportion of neurons with each response was compared with the expected $50 \%$ each by $\chi^{2}$ test, $p<0.001$.

${ }^{*} p<0.001$ (mean $\pm S E M$ ); firing rates were compared between both groups of dopamine responses (unpaired $t$ test).

state was unrelated to movement as opposed to bursts seen in normal monkeys (Kimura, 1990; Aldridge and Gilman, 1991; Kimura et al., 1996). Discharges of purely bursting type were rarely found. Nonbursty neurons exhibited slightly faster firing rates (putamen, $32.4 \pm 2.1 \mathrm{~Hz}$; caudate, $27.8 \pm 1.8 \mathrm{~Hz}$ ) than bursty neurons (putamen, $25.3 \pm 2.6 \mathrm{~Hz}$; caudate, $21.5 \pm 2 \mathrm{~Hz}$; $p<0.05$ unpaired $t$ tests). Because frequency changes that occurred in the transition to motor states did not differ between nonbursty and bursty neurons, the two groups will be considered together.

\section{Dopamine stimulation}

Although the transition to the On state (reversal of parkinsonism) was characterized by similar features in experiments with different doses of levodopa, data presented in this section correspond to 140 MSNs collected in experiments with dyskinetic doses of levodopa. In this section, activity changes are compared from the Off state to the onset of the On state in all neurons for correlation with normalization of mobility. Analysis of data in pools of cells showed rate increases from the Off to the On state: $29.9 \pm 1.7$ and $25.7 \pm 1.4 \mathrm{~Hz}$ to $33 \pm 2$ and $32.9 \pm 2.1 \mathrm{~Hz}$ in the putamen (73) and caudate (67), respectively. However, firing rates of individual MSNs changed in different directions and with magnitudes ranging from decreases of $44 \mathrm{~Hz}$ to increases of $47 \mathrm{~Hz}$ (more than $\pm 10 \mathrm{~Hz}$ in the majority of units; $p<0.0001$, ANOVAs for repeated measures followed by Bonferroni's tests). Only changes in which mean differences were above $z$ scores of $99 \%$ (see Materials and Methods) were computed. Notably, a higher proportion of MSNs exhibited increased firing rates after the onset of the On state instead of the expected restoration of the low rates present in normal conditions. The number of isolated units was consistent through the Off and On states, and thus silent units (not sampled) that became active during the On state were infrequent. In the putamen, $57.5 \%$, and in caudate, $70 \%$, of MSNs increased their firing rates, making a global $63.6 \%$ for all striatal MSNs $(p<0.0001)$ (Figs. $2 a-c, 3 a-c$, from Off to On states). Increases of firing rates in the On state averaged $14.5 \pm$ $1.3 \mathrm{~Hz}$ in the putamen and $14.9 \pm 1.5 \mathrm{~Hz}$ in the caudate.

In the opposite manner, a considerable number of MSNs exhibited decreased firing rates after the onset of the On state. Spontaneous loss of isolation of units that became silent after dopamine influx was rarely found (lost units and aborted experiments usually occurred with obvious movement). In the putamen, $38.4 \%$, and in caudate, $28.4 \%$, of MSNs decreased their firing rates, making $33.6 \%$ of all striatal MSNs $(p<0.0001)$ (Fig. $2 d-f$, $3 d-f$, from Off to On states). Decreases of firing rates in the On state averaged $13.4 \pm 1.7 \mathrm{~Hz}$ in the putamen and $11.6 \pm 2.7 \mathrm{~Hz}$ in 
the caudate. Unchanged firing rates of MSNs in the transition to the On state were infrequent in either the putamen (4\%) or caudate $(1.5 \%)$.

Increases and decreases of MSN activity in response to dopamine are here considered indicative of the excitatory action on $\mathrm{D}_{1}$ receptors and the inhibitory action on $\mathrm{D}_{2}$ receptors, respectively. These assumptions are based on most electrophysiology data from in vitro and in vivo studies (Kitai and Surmeier, 1993; Hernandez-Lopez et al., 2000; Cepeda et al., 2001; West and Grace, 2002; West et al., 2003). However, the alternative possibility of changes of activity in either direction depending on other factors than the activation of a certain receptor subtype, particularly in chronic pathological conditions, cannot be excluded with the available evidence and, particularly, the lack of data from identified neurons in behaving animals.

The proportion of MSNs with $\mathrm{D}_{1}(64 \%)$ and $\mathrm{D}_{2}$ (34\%) receptor-mediated responses did not match the predicted distribution of 50/50 in each output pathway (Table 1$)\left(p<0.001, \chi^{2}\right.$, observed vs expected). Knowing that we randomly sampled the units, the observed results correspond to the existent proportion of responses. Thus, $\mathrm{D}_{1}$ and $\mathrm{D}_{2}$ receptor-mediated responses were not distributed equally. Because there is no evidence for a distribution of MSNs different from an equivalent half in each output pathway, the explanation for these findings may lie in a less homogeneous receptor distribution and in receptor colocalization in the output pathways. According to studies in peptideidentified neurons of projection pathways (Surmeier et al., 1996), prevalent expression of $\mathrm{D}_{2}$-class receptors accounts for $86 \%$ of neurons expressing only the enkephalin peptide and projecting to the indirect pathway ( $\sim 38 \%$ of total), which form the majority of indirect projection neurons. Thus, the primary $\mathrm{D}_{2}$-class receptor group of MSNs corresponds to $\sim 33 \%$ of total MSNs. This proportion of indirect-pathway MSNs closely matches our results of $34 \%$ of neurons with dopamine response of decreased activity ( $\mathrm{D}_{2}$ receptors). The remaining $10-25 \%$ of indirect-pathway MSNs and a higher percentage of direct-pathway MSNs are reported to coexpress $\mathrm{D}_{1}$ and $\mathrm{D}_{2}$ receptors. Our group of $64 \%$ of the sampled MSNs responding to dopamine with activity increases includes all remaining units not responding with a net $D_{2}$ receptor-mediated effect, i.e., $M S N$ s with primary $D_{1}$-class receptors or with $D_{1} / D_{2}$ receptor colocalization. These data suggest that units with colocalization of receptors respond to dopamine with activity increases. According to the distribution of dopamine receptors (Surmeier et al., 1996), our group of $64 \%$ is distributed in $50 \%$ of neurons projecting to the direct pathway (primary $\mathrm{D}_{1}$ expression or $\mathrm{D}_{1} / \mathrm{D}_{2}$ coexpression), and the remaining $14 \%$ projecting to the indirect pathway $\left(D_{1} / D_{2}\right.$ coexpression in $10-25 \%$ MSNs). Therefore, not only MSNs expressing primarily $D_{1}$ receptors, but also those with $D_{1} / D_{2}$ receptor colocalization exhibit a net increase of activity in response to dopamine that is congruent with $\mathrm{D}_{1}$-mediated effects. These results show that activity increases in response to dopamine predominated across MSNs and likely involved a small proportion of units of the indirect output pathway.

\section{Drug-induced dyskinesias}

On average, firing rates of pooled data were similar in the On and the On-with-dyskinesias states, from $33 \pm 2$ and $32.9 \pm 2.1 \mathrm{~Hz}$ to $37 \pm 1.8$ and $33.4 \pm 2.3 \mathrm{~Hz}$ in the putamen and caudate, respectively. Analysis of rate changes in each neuron showed profound changes with two types of responses: (1) unidirectional change of rate, a progression of the same changes that occurred in the transition from the Off to the On state (Fig. 2); and (2) bidirectional
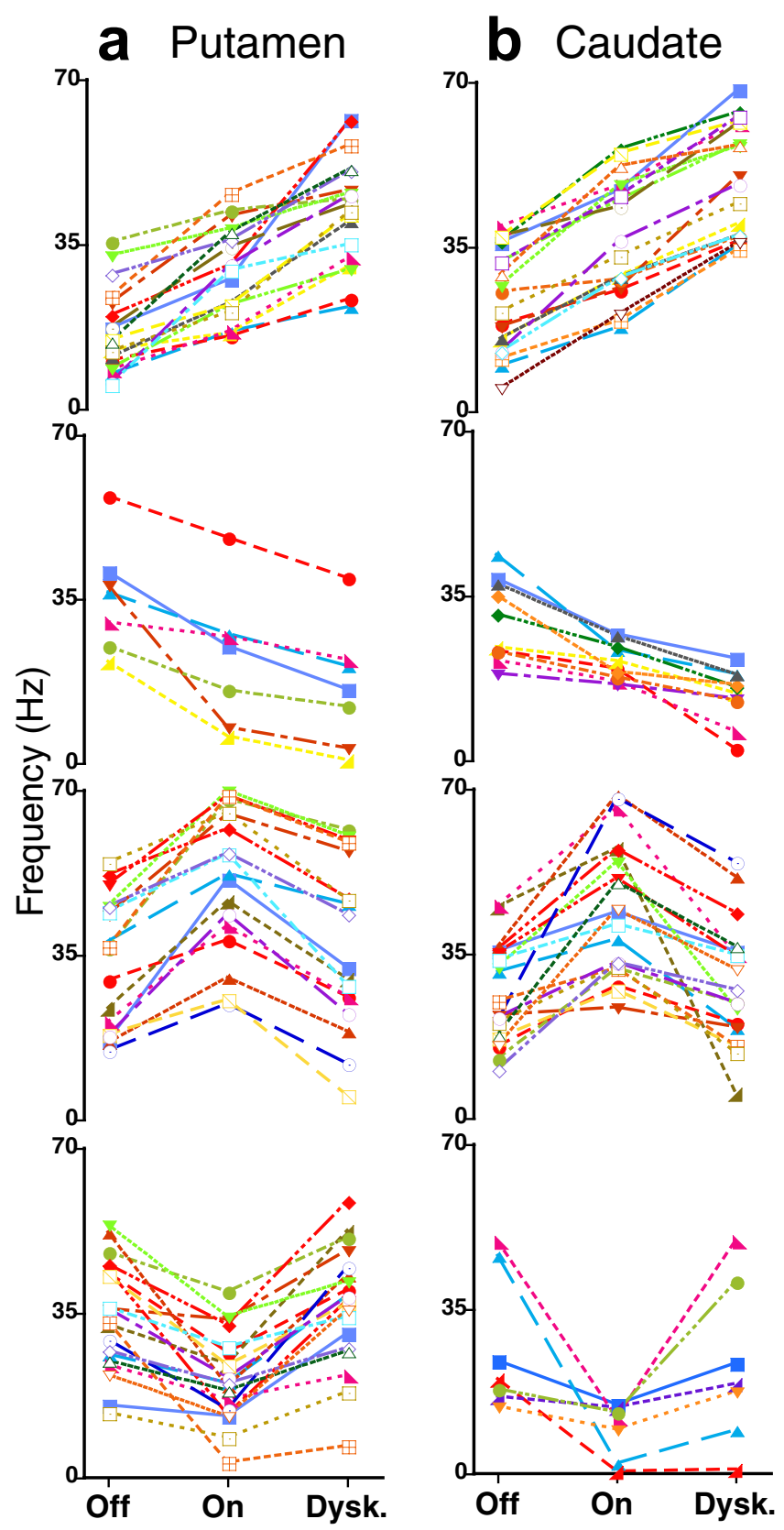

Figure 4. Changes of activity across MSNs with different responses. $\boldsymbol{a}, \boldsymbol{b}$, Firing frequencies of MSNs in the $(\boldsymbol{a})$ putamen and $(\boldsymbol{b})$ caudate grouped by type of responses from top to bottom: unidirectional increase, unidirectional decrease, bidirectional increase- decrease, and bidirectional decrease-increase. Each curve represents firing frequencies of a single neuron in the transition from Off to $0 \mathrm{n}$ and to $0 \mathrm{n}$-with-dyskinesias (Dysk.) states. Selected units for inclusion in the plots were 125 of total $(n=140)$ because of graphic limitations. Each single unit is represented in the graph by a curve of different color for clarity. All differences are significant at $p<0.01$ or lower (ANOVAs for repeated measures followed by Bonferroni's tests). Data points represent mean frequencies; error bars are not presented to visualize data points of the curves.

change of rate, an inversion of the changes that occurred in the transition from the Off to the On state (Fig. 3). Magnitudes of rate changes ( $p<0.0001$ for most differences) were similar as described above from the Off to the On state. Inversion of rate changes with dyskinesias led to a net offset of the On-state changes in some MSNs. Bidirectional responses were exhibited by both nonbursty and bursty neurons. Data of individual neurons were grouped by the type of response and are shown in Figure 4 for comparisons of distribution of units, range of 

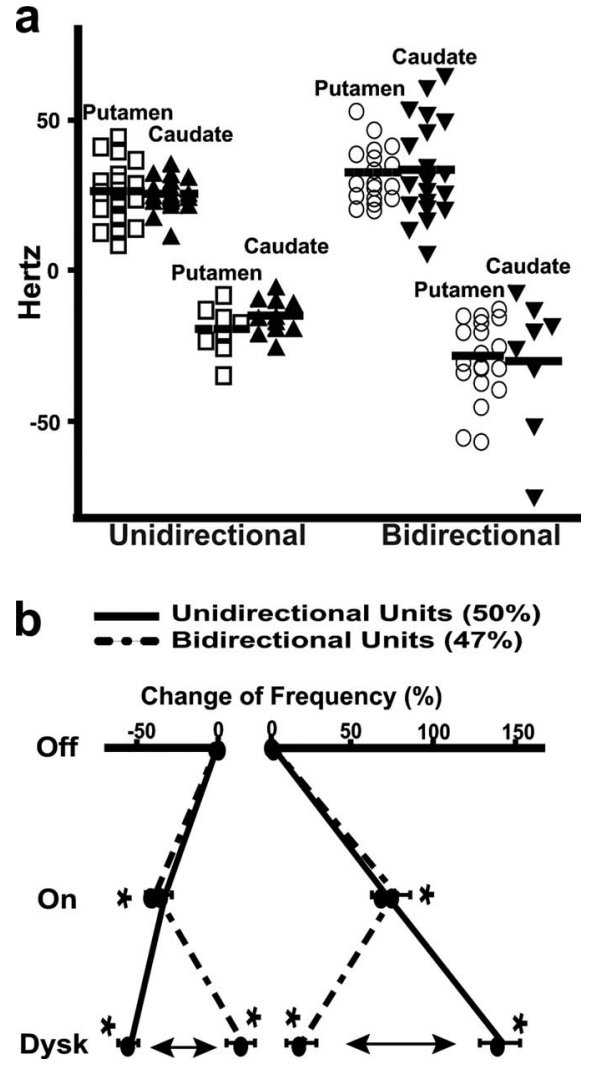

Figure 5. Similarity of frequency changes between the putamen and caudate, and the imbalance of MSN responses associated with dyskinesias. $\boldsymbol{a}$, Total magnitude of rate changes in each neuron in putamen (open symbols) and caudate (solid symbols). Units are grouped according to the direction of rate changes through the transition to motor states as unidirectional (increase-increase and decrease-decrease) and bidirectional (increase-decrease and decrease-increase). (orresponding groups from the putamen and caudate are plotted adjacently for comparison. The total change of rate in each unit was calculated from the algebraic addition (unidirectional groups) or subtraction (bidirectional groups) of rate differences between each motor state and the previous one. In bidirectional responses, total magnitudes of rate changes have a positive value for increase- decrease responses and a negative value for decrease-increase responses. Magnitudes of rate changes were indistinguishable between the putamen and caudate in each of the four group pairs (two-way ANOVA). $\boldsymbol{b}$, Averages of rate changes in all MSNs (putamen and caudate) grouped by their responses. The percentage of frequency change during $0 \mathrm{n}$ and $0 \mathrm{n}$-with-dyskinesias states with respect to the Off state (baseline, $0 \%$ change) is plotted in each group of responses (unidirectional, solid lines; bidirectional, dashed lines). Compared with the On state, dyskinesias are associated with a loss of balance within both MSN subpopulations (activity increase and decrease in the On state) because of large differences of firing rates as units exhibit bidirectional and unidirectional responses (arrows). ${ }^{*} p<0.001$ versus the previous state (ANOVAs for repeated measures followed by Bonferroni's tests; error bars indicate SEM).

changes across groups, and parallelism between the putamen and caudate.

MSNs with unidirectional and bidirectional responses were $43.8 \%$ and $52 \%$ in the putamen and $56.7 \%$ and $41.8 \%$ in caudate, respectively. Data of individual neurons collected in the putamen and caudate were indistinguishable, and comparisons of equivalent groups of responses in the two areas resulted in nonsignificant differences of rate changes (Fig. $5 a$ ). Thus, activity changes of MSNs in response to dopamine stimulation could be evaluated globally by grouping putamen and caudate units according to their response. In every type of response, averaged rates changed significantly from Off to On and from On to On-with-dyskinesias states $(p<0.0001)$ (Table 2). MSNs with unidirectional (50\%) and bidirectional $(47 \%)$ responses differed in their firing rates at baseline, $24.5 \pm 1.5$ and $31.4 \pm 1.5 \mathrm{~Hz}$, respectively $(p<0.003, t$
Table 2. Changes of MSN firing rates with different types of dopamine responses

\begin{tabular}{clll}
\hline & Off & On & Dysk \\
\hline Unidirectional responses (50\%) & & & \\
$\uparrow \uparrow(36 \%)$ & $21.1(1.6)$ & $33.3^{*}(2)$ & $44.1^{*}(2.2)$ \\
$\downarrow \downarrow(14 \%)$ & $33.6(2.6)$ & $22.6^{*}(2.2)$ & $16.5^{*}(2.3)$ \\
Bidirectional responses (47\%) & & & \\
$\uparrow \downarrow(27 \%)$ & $29.9(2)$ & $47.9^{*}(2.4)$ & $32.9^{*}(2.6)$ \\
$\downarrow \uparrow(20 \%)$ & $33.5(2.4)$ & $20^{*}(2)$ & $35.2^{*}(2.8)$ \\
\hline
\end{tabular}

Data derived from caudate and putamen MSNs pooled together. Units are grouped according to their response to dopamine stimulation as unidirectional [same direction of rate changes in the transition from the 0 off to the $0 \mathrm{n}$ and to the $0 \mathrm{n}$-with-dyskinesias (Dysk) states] and bidirectional (inversion of the previous rate changes in the transition to the 0n-with-dyskinesias state). The successive changes of rate from one state to another are indicated by the arrows, and the percentage of neurons showing such a response is given in parenthesis. In each group, the mean rate (in hertz) is provided for each motor state (Off, $0 \mathrm{n}$, and $0 \mathrm{n}$ with dyskinesias). Data are mean $\pm \mathrm{SEM}$.

${ }^{*} p<0.0001$ versus the previous state.

test). Assuming that bidirectional responses are specifically associated with dyskinesias (see below, On without dyskinesias), those percentages are consistent with the limited distribution and moderate intensity of dyskinesias in the experimental conditions of recordings. Comparisons of unidirectional and bidirectional responses show (1) higher firing rates in the Off state in units with bidirectional responses (Table 2), (2) similar changes from the Off to the On state (rate increases of 58 and $60 \%$ and rate decreases of 33 and $40 \%$ in units with unidirectional and bidirectional responses, respectively (Fig. 5b), and (3) a large asymmetry in the transition to the dyskinesias with further increases or decreases of frequency together with a return to nearly the same frequency as in the Off state in a high proportion of units. Dyskinesias were associated with marked changes of activity in some MSNs (increases and decreases of firing in unidirectional responses) but with suppression of changes in others (inversion of activity changes in bidirectional responses). Thus, these responses result in loss of equivalent actions of dopamine within MSN subpopulations in the On-with-dyskinesias state compared with the previous On state. Such a nonequivalence of dopamine responses is referred to as marked "imbalance" of MSN activity (Fig. 5b).

\section{On without dyskinesias}

To specifically relate frequency changes to dyskinesias as opposed to fluctuations of spontaneous neuronal activity during the On state, MSN activity was also recorded using lower doses of levodopa (nondyskinetic doses), with data collection as in experiments of higher doses. The selected lower doses did not elicit dyskinesias, although they induced a measurable and consistent On response (see Materials and Methods). Changes of firing rates during the transition from the Off to the On state were equivalent to those found in experiments of dyskinetic doses of levodopa. Analysis of data in pools of cells showed rate increases from the Off to the On state [from $22.8 \pm 2.8$ and $17.4 \pm 2.7 \mathrm{~Hz}$ to $25.7 \pm$ 3.3 and $28.1 \pm 3.7 \mathrm{~Hz}$ in the putamen (23) and caudate (25), respectively]. Analysis of rate individually in each neuron showed firing rates changing in different directions within the same range as in previous experiments from decreases of $47 \mathrm{~Hz}$ to increases of $39 \mathrm{~Hz}(p<0.0001)$ (Fig. 6). A high proportion of MSNs increased firing rates after the onset of the On state with nondyskinetic doses of levodopa (71\%, on average, of the striatum; $65 \%$, putamen; $76 \%$, caudate). Decreases of firing rates after the onset of the On state were found in $27 \%$ of MSNs (35\%, putamen; $20 \%$, caudate). The remaining $4 \%$ of caudate nucleus MSNs showed unchanged rates. Thus, data showed similar distributions of changes in experiments of both levodopa doses. During the interval corresponding to dyskinesias of previous experiments, fir- 


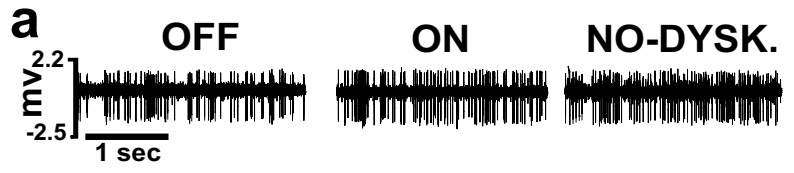

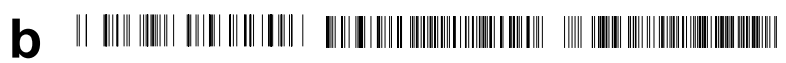
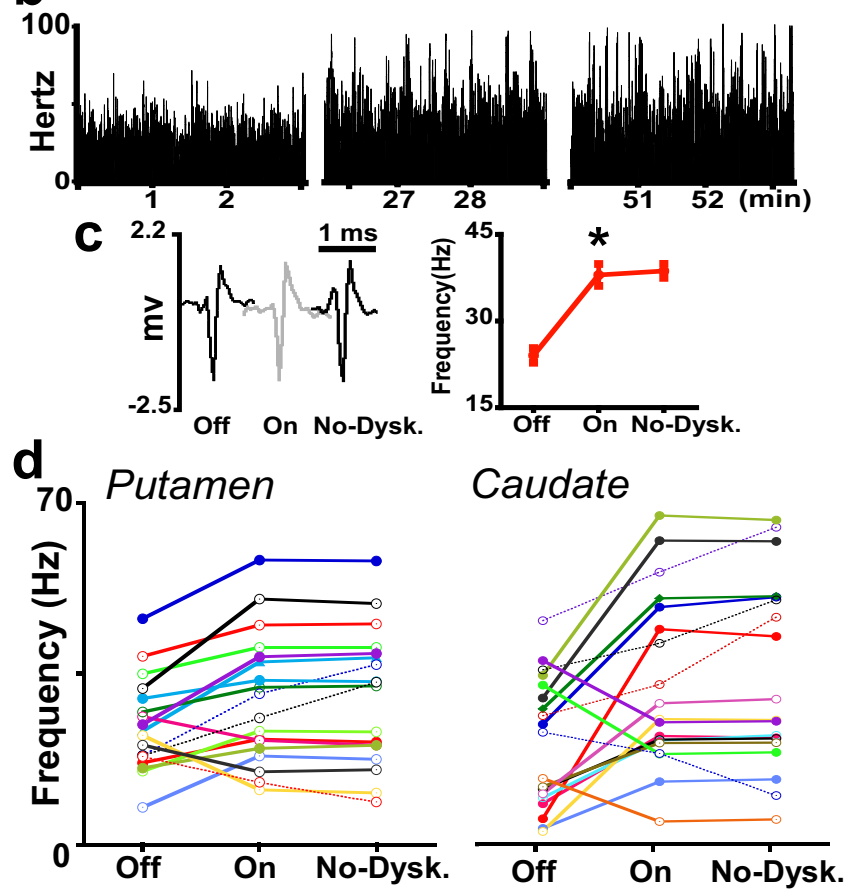

Figure 6. MSN activity changes in response to nondyskinetic doses of levodopa. Descriptions and conventions for $\boldsymbol{a}$ - $\boldsymbol{c}$ are the same as in Figure 2. $\boldsymbol{c}$, Right, The averaged rates are as follows: $24 \pm 1.3 \mathrm{~Hz}$ in $0 \mathrm{ff}, 38 \pm 1.9 \mathrm{~Hz}$ in $0 \mathrm{n}$, and $39 \pm 1.2 \mathrm{~Hz}$ in the On-without-dyskinesias (No-Dysk) state. This period is equivalent to the dyskinesias period of previous tests, but dyskinesias were absent in these tests with lower doses of levodopa. ${ }^{*} p<0.0001$ versus the previous state (error bars indicate SEM). $\boldsymbol{d}$, Activity changes of MSNs in the putamen and caudate as described in Figure 4. Rate changes from 0ff to 0 n states are significant at $p<0.01$ or lower. Rates remained unchanged (solid lines) or continued to change in the same direction (dashed lines) in the transition to the On-without-dyskinesias state (ANOVAs for repeated measures followed by Bonferroni's tests).

ing rates were maintained at the same level or continued to change in the same direction as at the onset of the On state (unidirectional responses) (Fig. $6 a-c$ ). On average, firing rates of pooled data did not change from the On state (putamen, $25.7 \pm$ $3.3 \mathrm{~Hz}$; caudate $28.1 \pm 3.7 \mathrm{~Hz}$ ) to the On-without-dyskinesias state (putamen, $26 \pm 3.4 \mathrm{~Hz}$; caudate, $29.1 \pm 3.9 \mathrm{~Hz}$ ). Analysis of rate individually in each neuron showed that rates continued to increase or decrease in 15\% $(p<0.0001)$ and remained unchanged in $85 \%$ of all MSNs (Fig. $6 d$ ). Therefore, in the Onwithout-dyskinesias state, MSNs respond with unidirectional or stable changes of rate. Inversion of rate changes was not found in these experiments of lower doses of levodopa and absence of dyskinesias during the On state.

\section{Discussion}

\section{Parkinsonian state}

This study demonstrates a markedly augmented firing rate of MSNs in the chronic parkinsonian setting. Spontaneous activity of MSNs averaging from 25 to $33 \mathrm{~Hz}$ consistently throughout striatal areas is markedly higher than previously reported in normal monkeys ( $<2 \mathrm{~Hz}$, usually silent units showing activity after stimulation) (Crutcher and DeLong, 1984; Alexander and De-
Long, 1985; Kimura, 1992; Aosaki et al., 1994). The most plausible cause for such differences is the advanced parkinsonism of the monkeys with extensive dopamine denervation. This is indicated precisely by the profound motor impairment of the animals because compensatory mechanisms may functionally interact with biochemical markers (Moratalla et al., 1992; Herrero et al., 1993; Di Monte et al., 2000). Our data are consistent with previous studies indicating increased excitatory drives on MSNs in dopamine-depleted animals (Oye et al., 1970; Ingham et al., 1998), and our results represent the MSN activity status of chronic PD.

In contrast to the hypothesized results, increased rates involved all MSNs and thus both striatal output pathways. Such rate increases across all MSNs imply a generalized mechanism, which does not distinguish between direct and indirect pathways, possibly related to exaggerated glutamatergic inputs from the cortex after loss of presynaptic dopamine regulation (Galarraga et al., 1987; Tang et al., 2001). Increased glutamatergic signaling in projection neurons in conditions of definite parkinsonism is also indicated by (1) changes at the synaptic level, such as loss of spines and contacts that may be related to hyperactivity of cortical afferents (Anglade et al., 1996; Ingham et al., 1998) or increase of postsynaptic excitability to glutamatergic inputs (Day et al., 2006; Shen et al., 2007), and (2) alterations of NMDA receptors with regard to subunit composition, cellular distribution, and phosphorylation status (Chase et al., 1998; Dunah et al., 2000). Our results provide evidence for changes of glutamatergic transmission after dopamine denervation leading to robust increase of MSN firing that contributes secondary dysregulation of striatal activity. These data have important implications for the functional model of the basal ganglia in PD. In contrast to the current notion of increased MSN activity in the indirect pathway and decreased MSN activity in the direct pathway, it is the increased output through both pathways that drive the abnormal signaling downstream from the striatum in PD. One might theorize that increased drives in both pathways result in an increase of opposing signals (GABAergic and glutamatergic) converging on the output nuclei [internal pallidum (GPi) and substantia nigra pars reticulata $(\mathrm{SNr})]$ that may produce altered and variable discharges and thereby cause parkinsonian symptoms. In fact, recordings of GPi in parkinsonian monkeys have shown altered patterns of discharge (irregularity, bursts, etc.) more consistently than increased rates (Raz et al., 2000; Heimer et al., 2006). Thus, the global increase of MSN discharges showed here seems to be the fundamental premise for a revision of current functional models.

\section{Dopamine action}

The initial response to dopamine leading to normalization of motor behavior was mediated by opposite effects across MSNs, thereby expressing the dual action of dopamine on $\mathrm{D}_{1}$ and $\mathrm{D}_{2}$ receptors. However, rate increases predominated throughout the striatum ( $64 \%$ of total MSNs) implying nonequivalent distribution of $D_{1}$ and $D_{2}$ responses in segregated pathways, as opposed to the expected results. Although without direct identification of MSN responses in each output pathway, our data indicate that $14 \%$ of units with activity increases are part of the total $50 \%$ projecting over the indirect pathway, i.e., MSNs with colocalization of $D_{1}$ and $D_{2}$ receptors, $\sim 10-25 \%$ (Surmeier et al., 1996). Therefore, neurons with receptor colocalization (14\% in the indirect and probably a higher proportion in the direct pathway) respond to dopamine with the activity increase typically produced by $D_{1}$-mediated effects. This may result from dominant $D_{1}$ 
receptor-mediated effects caused by upregulation after chronic denervation and dopaminergic treatment. However, upregulation of both receptor subtypes has been documented after dopaminergic lesions (Graham et al., 1993; Hurley et al., 2001; Cai et al., 2002), and the functional significance of such receptor changes remains uncertain. Our data suggesting a prevalent $D_{1}$ receptor function align with the development of behavioral supersensitivity after chronic $\mathrm{D}_{1}$-receptor agonist treatment (Gerfen, 2000). In addition, our results contrast with the net effect of dopamine in normal conditions (i.e., reduction of spontaneous firing of MSNs) (Kiyatkin and Rebec, 1999; Nicola et al., 2000), and thus dopamine responses after chronic lesions appear to reset toward a positive modulation ( $D_{1}$-mediated effects). The altered responsiveness of MSNs is probably related to the interaction of upregulated glutamatergic signaling (Cepeda et al., 1998; West et al., 2003), as derived from the major increases of activity found in the Off state.

The onset of the On state, compared with the dyskinesias period, was associated with discrete changes of MSN activities, which may correlate with similarly discrete changes found in connected basal ganglia loops (Papa et al., 1999; Heimer et al., 2006). However, the results presented here point to more heterogeneous dopamine responses than previously postulated in the indirect pathway (14\% units with activity increase) (Albin et al., 1989; DeLong, 1990). Whether this translates into something other than the expected net reduction of excitability in the indirect pathway remains to be addressed. However, the lack of equilibrated MSN responses between both pathways and the differential signaling within the indirect pathway suggest that dopamine responsiveness undergoes substantial changes after chronic lesions. These data are thus critical for revising functional models of drug effects in PD. Notably, by comparison with the Off state, after pronounced activity increases in both pathways, reversal of parkinsonian symptoms by dopaminergic stimulation occurs with an additional increase in $64 \%$ of units and a decrease in $34 \%$ of units. Therefore, it is the interplay of opposite drives on the output nuclei (GPi/SNr) rather than a certain level of activity in a single pathway that appears to mediate dopamine-induced behavioral changes. Overall, our data demonstrate that, after marked dopamine depletion with profound changes of striatal activity, responses to dopamine that reverse parkinsonian symptoms are more heterogeneous within the segregated pathways than predicted by the current model.

\section{Involuntary movements}

Involuntary movements are associated with inversions of dopamine responses across MSN subpopulations that result in a profound imbalance of discharges within each output pathway, as opposed to the predicted findings. Frequency changes that restored mobility earlier are amplified in some neurons and inverted in others, and such inversions were absent in the Onwithout-dyskinesias state. Our results show a progression of changes with increasing levels of dopamine going from unchanged rates and few unidirectional responses to more unidirectional plus bidirectional responses. Noticeably, inversion of responses occurs in MSNs with higher firing rates at baseline that may point to altered dopamine responses in units with more profound dysregulation of activity.

Dopamine receptor signaling mediates shifts of membrane excitability (Wilson and Kawaguchi, 1996; Wickens and Wilson, 1998; Kitano et al., 2002; West et al., 2003) that may cause bidirectional responses of MSNs during increasing dopamine inputs. The predominant activation of a receptor subtype in neurons with $D_{1} / D_{2}$ receptor colocalization may be construed as the basis for such responses (Chiodo and Berger, 1986; Hu and Wang, 1988; Tecuapetla et al., 2007) because, as in general agonistreceptor interaction, the activation of receptors may extend to the less sensitive receptors with increasing dopamine levels. Yet, dopamine may have differential effects on the same receptor subtype depending on the level of stimulation (Shen et al., 1992). However, the role of the increased glutamate transmission after dopamine depletion may be central to bidirectional responses. Electrophysiology studies in rodents have shown the significance of glutamatergic hyperactivity in the mechanisms of dyskinesias (Calabresi et al., 2000; Picconi et al., 2002; Gubellini et al., 2006). In hemiparkinsonian rats, normalization of motor behavior without inducing dyskinesias requires reversal of the abnormal glutamatergic drive (Picconi et al., 2004; Centonze et al., 2005). In addition, glutamate-mediated mechanisms such as long-term potentiation and depression in MSNs show altered dopamine regulation in denervated and dyskinetic rats (Centonze et al., 2001; Picconi et al., 2003; Gubellini et al., 2004). Most physiologic and biochemical data point to an increased glutamatergic function after chronic levodopa treatment. The findings of bidirectional responses in MSNs with higher baseline frequencies further support mechanisms related to glutamatergic hyperactivity.

Our data at the basal ganglia circuit level indicate that inversion of dopamine responses occurs in segregated MSNs of the direct and indirect output pathways. Therefore, both pathways are involved in the mechanisms of involuntary movements, and they cannot be modeled with changes affecting only a single loop of the circuitry. It has been hypothesized (Wichmann and DeLong, 1996; Mink, 2003) that an imbalance of striatal activity can generate involuntary movements by facilitating mobility without appropriate inhibition of motor routines. Our direct recordings of single-MSN activity in parkinsonian monkeys show that an imbalance of activity within each output pathway is caused by inversion of dopamine responses under a secondarily dysregulated status of MSN firing after chronic dopamine depletion and levodopa treatment. Thus, targeting the glutamate system or participating signaling mechanisms to restore an equilibrated activity in striatal projection neurons may be key to the long-term therapy of PD.

\section{References}

Albin RL, Young AB, Penney JB (1989) The functional anatomy of basal ganglia disorders. Trends Neurosci 12:366-375.

Aldridge JW, Gilman S (1991) The temporal structure of spike trains in the primate basal ganglia: afferent regulation of bursting demonstrated with precentral cerebral cortical ablation. Brain Res 543:123-138.

Alexander GE, DeLong MR (1985) Microstimulation of the primate neostriatum. II. Somatotopic organization of striatal microexcitable zones and their relation to neuronal response properties. J Neurophysiol 53:1417-1430.

Anglade P, Mouatt-Prigent A, Agid Y, Hirsch E (1996) Synaptic plasticity in the caudate nucleus of patients with Parkinson's disease. Neurodegeneration 5:121-128.

Aosaki T, Tsubokawa H, Ishida A, Watanabe K, Graybiel AM, Kimura M (1994) Responses of tonically active neurons in the primate's striatum undergo systematic changes during behavioral sensorimotor conditioning. J Neurosci 14:3969-3984.

Barnes TD, Kubota Y, Hu D, Jin DZ, Graybiel AM (2005) Activity of striatal neurons reflects dynamic encoding and recoding of procedural memories. Nature 437:1158-1161.

Cai G, Wang HY, Friedman E (2002) Increased dopamine receptor signaling and dopamine receptor- $G$ protein coupling in denervated striatum. J Pharmacol Exp Ther 302:1105-1112.

Calabresi P, Centonze D, Gubellini P, Marfia GA, Pisani A, Sancesario G, 
Bernardi G (2000) Synaptic transmission in the striatum: from plasticity to neurodegeneration. Prog Neurobiol 61:231-265.

Centonze D, Picconi B, Gubellini P, Bernardi G, Calabresi P (2001) Dopaminergic control of synaptic plasticity in the dorsal striatum. Eur J Neurosci 13:1071-1077.

Centonze D, Gubellini P, Rossi S, Picconi B, Pisani A, Bernardi G, Calabresi P, Baunez C (2005) Subthalamic nucleus lesion reverses motor abnormalities and striatal glutamatergic overactivity in experimental parkinsonism. Neuroscience 133:831-840.

Cepeda C, Colwell CS, Itri JN, Chandler SH, Levine MS (1998) Dopaminergic modulation of NMDA-induced whole cell currents in neostriatal neurons in slices: contribution of calcium conductances. J Neurophysiol 79:82-94.

Cepeda C, Hurst RS, Altemus KL, Flores-Hernández J, Calvert CR, Jokel ES, Grandy DK, Low MJ, Rubinstein M, Ariano MA, Levine MS (2001) Facilitated glutamatergic transmission in the striatum of D2 dopamine receptor-deficient mice. J Neurophysiol 85:659-670.

Chase TN, Engber TM, Mouradian MM (1996) Contribution of dopaminergic and glutamatergic mechanisms to the pathogenesis of motor response complications in Parkinson's disease. Adv Neurol 69:497-501.

Chase TN, Oh JD, Blanchet PJ (1998) Neostriatal mechanisms in Parkinson's disease. Neurology 51:S30-S35.

Chiodo LA, Berger TW (1986) Interactions between dopamine and amino acid-induced excitation and inhibition in the striatum. Brain Res 375:198-203.

Costa RM, Lin SC, Sotnikova TD, Cyr M, Gainetdinov RR, Caron MG, Nicolelis MA (2006) Rapid alterations in corticostriatal ensemble coordination during acute dopamine-dependent motor dysfunction. Neuron 52:359-369.

Crutcher MD, DeLong MR (1984) Single cell studies of the primate putamen. I. Functional organization. Exp Brain Res 53:233-243.

Day M, Wang Z, Ding J, An X, Ingham CA, Shering AF, Wokosin D, Ilijic E, Sun Z, Sampson AR, Mugnaini E, Deutch AY, Sesack SR, Arbuthnott GW, Surmeier DJ (2006) Selective elimination of glutamatergic synapses on striatopallidal neurons in Parkinson disease models. Nat Neurosci 9:251-259.

DeLong MR (1990) Primate models of movement disorders of basal ganglia origin. Trends Neurosci 13:281-285.

Di Monte DA, McCormack A, Petzinger G, Janson AM, Quik M, Langston WJ (2000) Relationship among nigrostriatal denervation, parkinsonism, and dyskinesias in the MPTP primate model. Mov Disord 15:459-466.

Dunah AW, Wang Y, Yasuda RP, Kameyama K, Huganir RL, Wolfe BB, Standaert DG (2000) Alterations in subunit expression, composition, and phosphorylation of striatal N-methyl-D-aspartate glutamate receptors in a rat 6-hydroxydopamine model of Parkinson's disease. Mol Pharmacol 57:342-352.

Galarraga E, Bargas J, Martínez-Fong D, Aceves J (1987) Spontaneous synaptic potentials in dopamine-denervated neostriatal neurons. Neurosci Lett 81:351-355.

Gerfen CR (1992) The neostriatal mosaic: multiple levels of compartmental organization. Trends Neurosci 15:133-139.

Gerfen CR (2000) Molecular effects of dopamine on striatal-projection pathways. Trends Neurosci 23:S64-70.

Graham WC, Sambrook MA, Crossman AR (1993) Differential effect of chronic dopaminergic treatment on dopamine D1 and D2 receptors in the monkey brain in MPTP-induced parkinsonism. Brain Res 602:290-303.

Graybiel AM, Aosaki T, Flaherty AW, Kimura M (1994) The basal ganglia and adaptive motor control. Science 265:1826-1831.

Gubellini P, Picconi B, Bari M, Battista N, Calabresi P, Centonze D, Bernardi G, Finazzi-Agrò A, Maccarrone M (2002) Experimental parkinsonism alters endocannabinoid degradation: implications for striatal glutamatergic transmission. J Neurosci 22:6900-6907.

Gubellini P, Pisani A, Centonze D, Bernardi G, Calabresi P (2004) Metabotropic glutamate receptors and striatal synaptic plasticity: implications for neurological diseases. Prog Neurobiol 74:271-300.

Gubellini P, Eusebio A, Oueslati A, Melon C, Kerkerian-Le Goff L, Salin P (2006) Chronic high-frequency stimulation of the subthalamic nucleus and L-DOPA treatment in experimental parkinsonism: effects on motor behaviour and striatal glutamate transmission. Eur J Neurosci 24:1802-1814.

Heimer G, Rivlin-Etzion M, Bar-Gad I, Goldberg JA, Haber SN, Bergman H
(2006) Dopamine replacement therapy does not restore the full spectrum of normal pallidal activity in the 1-methyl-4-phenyl-1,2,3,6-tetrahydropyridine primate model of Parkinsonism. J Neurosci 26:8101-8114.

Hernandez-Lopez S, Tkatch T, Perez-Garci E, Galarraga E, Bargas J, Hamm H, Surmeier DJ (2000) D2 dopamine receptors in striatal medium spiny neurons reduce L-type $\mathrm{Ca}^{2+}$ currents and excitability via a novel PLC[beta] 1-IP3-calcineurin-signaling cascade. J Neurosci 20:8987-8995.

Herrero MT, Hirsch EC, Kastner A, Ruberg M, Luquin MR, Laguna J, JavoyAgid F, Obeso JA, Agid Y (1993) Does neuromelanin contribute to the vulnerability of catecholaminergic neurons in monkeys intoxicated with MPTP? Neuroscience 56:499-511.

Hu XT, Wang RY (1988) Comparison of effects of D-1 and D-2 dopamine receptor agonists on neurons in the rat caudate putamen: an electrophysiological study. J Neurosci 8:4340-4348.

Hurley MJ, Mash DC, Jenner P (2001) Dopamine D(1) receptor expression in human basal ganglia and changes in Parkinson's disease. Brain Res Mol Brain Res 87:271-279.

Ingham CA, Hood SH, Taggart P, Arbuthnott GW (1998) Plasticity of synapses in the rat neostriatum after unilateral lesion of the nigrostriatal dopaminergic pathway. J Neurosci 18:4732-4743.

Kaneoke Y, Vitek JL (1996) Burst and oscillation as disparate neuronal properties. J Neurosci Methods 68:211-223.

Kimura M (1986) The role of primate putamen neurons in the association of sensory stimuli with movement. Neurosci Res 3:436-443.

Kimura M (1990) Behaviorally contingent property of movement-related activity of the primate putamen. J Neurophysiol 63:1277-1296.

Kimura M (1992) Behavioral modulation of sensory responses of primate putamen neurons. Brain Res 578:204-214.

Kimura M, Kato M, Shimazaki H, Watanabe K, Matsumoto N (1996) Neural information transferred from the putamen to the globus pallidus during learned movement in the monkey. J Neurophysiol 76:3771-3786.

Kitai ST, Surmeier DJ (1993) Cholinergic and dopaminergic modulation of potassium conductances in neostriatal neurons. Adv Neurol 60:40-52.

Kitano K, Câteau H, Kaneda K, Nambu A, Takada M, Fukai T (2002) Twostate membrane potential transitions of striatal spiny neurons as evidenced by numerical simulations and electrophysiological recordings in awake monkeys. J Neurosci 22:RC230(1-6).

Kiyatkin EA, Rebec GV (1999) Striatal neuronal activity and responsiveness to dopamine and glutamate after selective blockade of D1 and D2 dopamine receptors in freely moving rats. J Neurosci 19:3594-3609.

Kreitzer AC, Malenka RC (2007) Endocannabinoid-mediated rescue of striatal LTD and motor deficits in Parkinson's disease models. Nature 445:643-647.

Mink JW (2003) The basal ganglia and involuntary movements: impaired inhibition of competing motor patterns. Arch Neurol 60:1365-1368.

Moratalla R, Quinn B, DeLanney LE, Irwin I, Langston JW, Graybiel AM (1992) Differential vulnerability of primate caudate-putamen and striosome-matrix dopamine systems to the neurotoxic effects of 1-methyl-4-phenyl-1,2,3,6-tetrahydropyridine. Proc Natl Acad Sci U S A 89:3859-3863.

Nicola SM, Surmeier J, Malenka RC (2000) Dopaminergic modulation of neuronal excitability in the striatum and nucleus accumbens. Annu Rev Neurosci 23:185-215.

Onn SP, West AR, Grace AA (2000) Dopamine-mediated regulation of striatal neuronal and network interactions. Trends Neurosci 23:S48-56.

Oye C, Bouchard R, Boucher R, Poirier LJ (1970) Spontaneous activity of the putamen after chronic interruption of the dopaminergic pathway: effect of L-dopa. J Pharmacol Exp Ther 175:700-708.

Papa SM, Chase TN (1996) Levodopa-induced dyskinesias improved by a glutamate antagonist in Parkinsonian monkeys. Ann Neurol 39:574-578.

Papa SM, Desimone R, Fiorani M, Oldfield EH (1999) Internal globus pallidus discharge is nearly suppressed during levodopa-induced dyskinesias. Ann Neurol 46:732-738.

Picconi B, Pisani A, Centonze D, Battaglia G, Storto M, Nicoletti F, Bernardi G, Calabresi P (2002) Striatal metabotropic glutamate receptor function following experimental parkinsonism and chronic levodopa treatment. Brain 125:2635-2645.

Picconi B, Centonze D, Håkansson K, Bernardi G, Greengard P, Fisone G, Cenci MA, Calabresi P (2003) Loss of bidirectional striatal synaptic plasticity in L-DOPA-induced dyskinesia. Nat Neurosci 6:501-506.

Picconi B, Centonze D, Rossi S, Bernardi G, Calabresi P (2004) Therapeutic 
doses of L-dopa reverse hypersensitivity of corticostriatal D2-dopamine receptors and glutamatergic overactivity in experimental parkinsonism. Brain 127:1661-1669.

Plenz D, Kitai ST (1998) Up and down states in striatal medium spiny neurons simultaneously recorded with spontaneous activity in fast-spiking interneurons studied in cortex-striatum-substantia nigra organotypic cultures. J Neurosci 18:266-283.

Raz A, Feingold A, Zelanskaya V, Vaadia E, Bergman H (1996) Neuronal synchronization of tonically active neurons in the striatum of normal and parkinsonian primates. J Neurophysiol 76:2083-2088.

Raz A, Vaadia E, Bergman H (2000) Firing patterns and correlations of spontaneous discharge of pallidal neurons in the normal and the tremulous 1-methyl-4-phenyl-1,2,3,6-tetrahydropyridine vervet model of parkinsonism. J Neurosci 20:8559-8571.

Shen RY, Asdourian D, Chiodo LA (1992) Microiontophoretic studies of the effects of D-1 and D-2 receptor agonists on type I caudate nucleus neurons: lack of synergistic interaction. Synapse 11:319-329.

Shen W, Tian X, Day M, Ulrich S, Tkatch T, Nathanson NM, Surmeier DJ (2007) Cholinergic modulation of Kir 2 channels selectively elevates dendritic excitability in striatopallidal neurons. Nat Neurosci 10:1458-1466.

Surmeier DJ, Bargas J, Hemmings HC Jr, Nairn AC, Greengard P (1995) Modulation of calcium currents by a D1 dopaminergic protein kinase/ phosphatase cascade in rat neostriatal neurons. Neuron 14:385-397.

Surmeier DJ, Song WJ, Yan Z (1996) Coordinated expression of dopamine receptors in neostriatal medium spiny neurons. J Neurosci $16: 6579-6591$.
Tang K, Low MJ, Grandy DK, Lovinger DM (2001) Dopamine-dependent synaptic plasticity in striatum during in vivo development. Proc Natl Acad Sci U S A 98:1255-1260.

Tecuapetla F, Carrillo-Reid L, Bargas J, Galarraga E (2007) Dopaminergic modulation of short-term synaptic plasticity at striatal inhibitory synapses. Proc Natl Acad Sci U S A 104:10258-10263.

Wang Z, Kai L, Day M, Ronesi J, Yin HH, Ding J, Tkatch T, Lovinger DM, Surmeier DJ (2006) Dopaminergic control of corticostriatal long-term synaptic depression in medium spiny neurons is mediated by cholinergic interneurons. Neuron 50:443-452.

West AR, Grace AA (2002) Opposite influences of endogenous dopamine D1 and D2 receptor activation on activity states and electrophysiological properties of striatal neurons: studies combining in vivo intracellular recordings and reverse microdialysis. J Neurosci 22:294-304.

West AR, Floresco SB, Charara A, Rosenkranz JA, Grace AA (2003) Electrophysiological interactions between striatal glutamatergic and dopaminergic systems. Ann N Y Acad Sci 1003:53-74.

Wichmann T, DeLong MR (1996) Functional and pathophysiological models of the basal ganglia. Curr Opin Neurobiol 6:751-758.

Wickens JR, Wilson CJ (1998) Regulation of action-potential firing in spiny neurons of the rat neostriatum in vivo. J Neurophysiol 79:2358-2364.

Wilson CJ, Kawaguchi Y (1996) The origins of two-state spontaneous membrane potential fluctuations of neostriatal spiny neurons. J Neurosci 16:2397-2410.

Winters WD, Kado RT, Adey WR (1969) A stereotaxic brain atlas for Macaca nemestrina. Berkeley: University of California. 